University of Nebraska - Lincoln

DigitalCommons@University of Nebraska - Lincoln

June 2006

\title{
Selenoproteins in parasites
}

\author{
Gustavo Salinas \\ Universidad de la República, Montevideo, Uruguay
}

Alexei V. Lobanov

University of Nebraska-Lincoln

Vadim N. Gladyshev

University of Nebraska-Lincoln, vgladyshev@rics.bwh.harvard.edu

Follow this and additional works at: https://digitalcommons.unl.edu/biochemgladyshev

Part of the Biochemistry, Biophysics, and Structural Biology Commons

Salinas, Gustavo; Lobanov, Alexei V.; and Gladyshev, Vadim N., "Selenoproteins in parasites" (2006). Vadim Gladyshev Publications. 40.

https://digitalcommons.unl.edu/biochemgladyshev/40

This Article is brought to you for free and open access by the Biochemistry, Department of at DigitalCommons@University of Nebraska - Lincoln. It has been accepted for inclusion in Vadim Gladyshev Publications by an authorized administrator of DigitalCommons@University of Nebraska - Lincoln. 
Published in Selenium: Its molecular biology and role in human health, Second Edition, edited by Dolph L. Hatfield, Marla J. Berry, and Vadim N. Gladyshev. Springer Science+Business Media LLC, 2006. Pages 355-366. Copyright (C) 2006 Springer Science+Business Media LLC. Used by permission.

Chapter 31

\title{
Selenoproteins in parasites
}

\author{
Gustavo Salinas \\ Cátedra de Inmunología, Facultad de Química-Facultad de Ciencias, Universidad de la \\ República. Instituto de Higiene, Avda. A. Navarro 3051, Montevideo, CP 11600, Uruguay \\ Alexey V. Lobanov \& Vadim N. Gladyshev \\ Department of Biochemistry, University of Nebraska-Lincoln, Lincoln, NE 68588-0664, USA
}

\begin{abstract}
Summary: Parasites, which cause an enormous burden in the population of the third world, are a diverse group of organisms, many of which are sensitive to oxidative stress imposed by their hosts. In recent years, several selenoprotein families, some with antioxidant properties, have been described and characterized in metazoan parasites. Glutathione peroxidase and thioredoxin glutathione reductase (TGR) appear to be essential selenoproteins in flatworms (phylum Platyhelminthes). TGR is the single enzyme that provides reducing equivalents to both thioredoxin and glutathione pathways, in contrast to hosts, which evolve parallel pathways. In roundworms (phylum Nematoda), selenoproteins have recently been described, revealing species differences in the Sec/Cys protein sets and the presence of an unusual SECIS element. Plasmodium sp, one of the most important protozoan parasites that affect humans, also decode Sec. The selenoprotein families encoded by Plasmodial genomes have neither Sec nor Cys homologs in their hosts, raising the possibility that targeting their selenoproteomes may provide new treatment strategies.
\end{abstract}

\section{Introduction}

Although significant research efforts have been made to study selenoproteins and selenocysteine insertion systems in humans and various model organisms, little has been reported in the literature regarding the utilization of selenium in eukaryotic parasitic organisms. This chapter focuses on the progress made in the characterization of selenoenzyme families in flatworms, the recent advances in the synthesis and utilization of selenoproteins in roundworms and protozoan parasites, and discusses why selenoproteins of platyhelminths and plasmodia may represent interesting targets for chemo- or immune-prophylaxis. 
Parasites: diverse organisms that face similar oxidative stress challenges Parasites live at least part of their lifecycle inside another organism (the host), which they exploit for their own survival and reproduction. This definition includes different types of infectious agents (viruses, bacteria, fungi, protozoa, helminths). However, for historical reasons, the term is most often reserved for 'protozoa' and 'helminths' organisms. Indeed, parasitology was identified as a separate research field during the exploration of the tropics and the establishment of 'tropical medicine' [1]. Both 'protozoa' and 'helminths' also include free-living organisms, and neither 'protozoa' nor 'helminths' are monophyletic; on the contrary, both groups are represented by highly divergent phyla. Nonetheless, this historical classification is not useless. These two groups of parasites are very different: protozoan are unicellular protists, which multiply quickly within the host, and are, in most cases, intracellular in habitat; in contrast, helminths are metazoan organisms with complex multicellular organization (with nervous system and reproductive organs), which undergo complex metamorphoses and migrations within the host. Table 1 presents the main features of the major human parasitic infections.

In spite of the diversity of parasites, all face similar biological problems that relate to their parasitic lifestyle. Among them, the neutralization of the effector mechanisms deployed by the host immune system is of paramount importance. Resident macrophages and inflammatory-site phagocytic leukocytes (mostly neutrophils, but also monocytes and eosinophils, depending of the type of infection) are cells equipped to kill foreign organisms. They possess an oxidase system located in their plasma membrane, which becomes activated upon certain stimuli, for example, by interaction of cell receptors with antibodies bound to the foreign organism or with parasite molecular motifs (Figure la) [3]. Subsequently, 'respiratory burst' (increase in oxygen uptake not linked to respiration) takes place and produces superoxide anion and additional reactive oxygen species (ROS) [4]. Large amounts of nitric oxide ('NO) are also produced by macrophages (and to a lesser extent by neutrophils) activated by a variety of immunological stimuli, such as y-interferon and tumor necrosis factor. 'NO reacts with superoxide to produce peroxynitrite and other reactive nitrogen species (RNS) (Figure 1b) [5]. In addition, activated neutrophils and eosinophils release myeloperoxidase and eosinophil peroxidase, respectively, that catalyze the conversion of hydrogen peroxide and halides into hypohalous acids that are powerful oxidants and can form further damaging species [4].

Collectively, ROS and RNS are powerful oxidants and nitrating species: they can inactivate enzymes and initiate the process of lipid peroxidation and nitration, which leads to radical chain reactions that further damage membranes, nucleic acids and proteins (Figure 1c). These processes (and an additional arsenal of the host effector cells, such as hydrolytic enzymes) may ultimately lead to killing parasitic organisms. Yet, well-adapted parasites cope

Table 1. Major human parasites (Source: [2])

\section{Protozoan parasites ${ }^{\mathrm{b}}$}

Species (Disease)

Plasmodium sp

$\left(\right.$ Malaria $\left.^{c}\right)$

Trypanosoma brucei

(sleeping sickness ${ }^{\mathrm{d}}$ )

Trypanosoma cruzi

(Chagas disease $\left.{ }^{\mathrm{e}}\right)$

Leishmania sp

$\left(\right.$ Leishmaniasis $\left.^{\mathrm{f}}\right)$

\section{Helminths parasites}

\section{Species/Disease}

Schistosoma sp

(Schistosomiasis or bilharzia ${ }^{\mathrm{g}}$

Onchocerca volvulus

(Onchocerciasis or river blindness ${ }^{\mathrm{h}}$ )

\section{Filariidae family}

(Lymphatic filariasisi ${ }^{\mathrm{i}}$

Phylum

Apicomplexa

Kinetoplastida

Kinetoplastida

Kinetoplastida

$0 / 649,000$

$59,000 / 2,357,000$

Phylum

Plathyhelminthes

Death per year/DALYs

$15,000 / 1,760,000$

${ }^{a}$ DALYs: Disability Adjusted Life Years (the number of healthy years of life lost due to premature death and disability).

b Protozoan parasites include many diverse phyla, among them Apicomplexa and Kinetoplastida.

${ }^{\mathrm{c}}$ Distribution: mainly confined to poorer tropical areas of Africa, Asia and Latin America. More than $90 \%$ of malaria cases and the great majority of malaria deaths occur in tropical Africa. Plasmodium falciparum is the main cause of severe clinical malaria and death.

${ }^{\mathrm{d}}$ Distribution: 36 countries in sub-Saharan Africa

${ }^{\text {e }}$ Distribution: Latin America

${ }^{\mathrm{f}}$ Distribution: Endemic in 88 countries on 4 continents. Two forms of the disease: cutaneous (caused by Leishmania major), and visceral (caused by L. donovani)

${ }^{\mathrm{g}}$ Helminth parasites are contained in three phyla: Nematoda (roundworms), Platyhelminthes (flatworms) and Acantocephala (spiny-headed worms). Helminth infections are rarely fatal, but pose an enormous burden to human population in the tropics Distribution: endemic in 74 developing countries with more than $80 \%$ of infected people living in subSaharan Africa

${ }^{\text {h }}$ Distribution: 35 countries in total. 28 in tropical Africa, where $99 \%$ of infected people live. Isolated foci in Latin America and Yemen.

${ }^{i}$ Distribution: Endemic in over 80 countries in Africa, Asia, South and Central America and the Pacific Islands. Three species are of significance, Wuchereria bancrofti, Brugia malayi, and Brugia timori. 


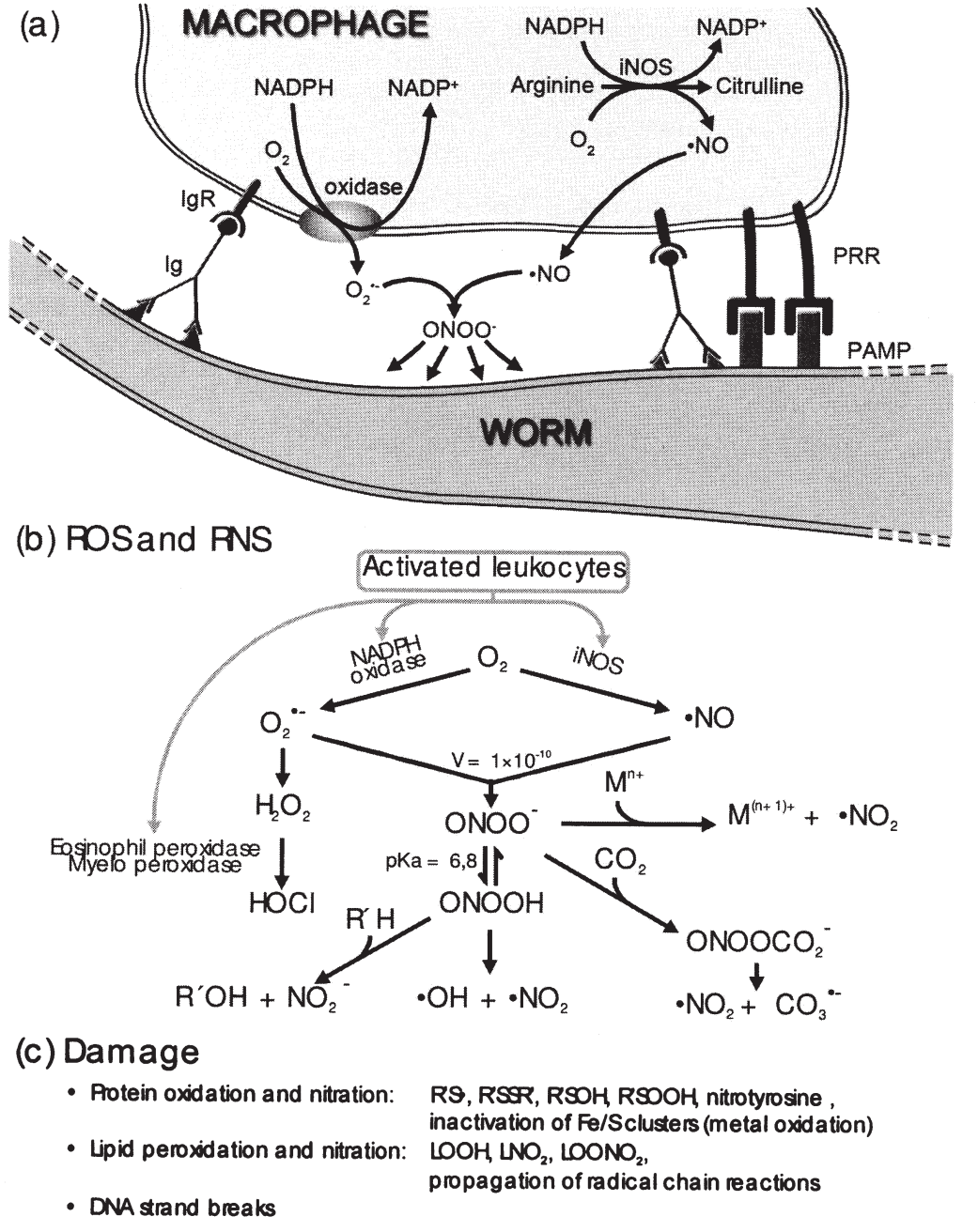

(d) Enzymatic defenses

\begin{tabular}{|c|c|c|}
\hline SOD. & dismutation of superoxide & $2 \mathrm{O}_{2}^{--} \rightarrow \mathrm{O}_{2}+\mathrm{H}_{2} \mathrm{O}_{2}$ \\
\hline GPX I & reduction of peroxides & $\mathrm{H}_{2} \mathrm{O}_{2} \rightarrow \mathrm{H}_{2} \mathrm{O}$ \\
\hline $\mathbb{R P}\}$ & 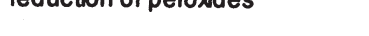 & $\mathrm{ROOH} \rightarrow \mathrm{ROH}$ \\
\hline PX: & reduction of sulfenic acid & $\mathrm{RSOH} \rightarrow \mathrm{RSH}$ \\
\hline $\begin{array}{l}\text { Some TPX: } \\
\text { Pepair enza }\end{array}$ & $\begin{array}{l}\text { reduction of peroxynitrite } \\
\text { mes: }\end{array}$ & $\mathrm{ONOOH} \rightarrow \mathrm{NO}_{2}$ \\
\hline & Tx reduction of protein disulfides & $R S R \rightarrow R S H$ \\
\hline & 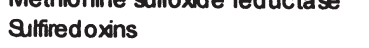 & $\mathrm{RSOOH} \rightarrow \mathrm{RSOH}$ \\
\hline
\end{tabular}

with the oxidative stress imposed by the host's immune response by a series of cellular chemicals and antioxidant enzymes that directly neutralize ROS and RNS (Figure Id), and constitute important model organisms to study antioxidant defense. Several antioxidant enzymes found in parasites belong to selenoprotein families.

Glutathione peroxidase: the first selenoenzyme described in parasites Glutathione peroxidase was the first selenoenzyme to be characterized from a parasite. A cDNA from the platyhelminth Schistosoma mansoni encoding a GPx with a TGA in-frame at the active site was cloned in the early 1990s [6]. The protein encoded by this gene has biochemical properties similar to mammalian phospholipid hydroperoxide glutathione peroxidase (PHGPx); its activity being higher with phosphatidyl choline hydroperoxide and other phospholipid hydroperoxides than with hydroperoxide substrates, such as cumene hydroperoxide and hydrogen peroxide [7]. GPx and superoxide dismutase, another antioxidant enzyme, co-localize in the tegument and gut epithelium of adult worms, which are the exposed interfaces of the parasite towards the host [8]. Additional evidence suggests that antioxidant enzymes, and GPx in particular, are vital for ROS neutralization and parasite survival within the host. Indeed, expression of GPx is developmentally regulated,

Figure 1. Reactive oxygen and nitrogen species generated by the host immune response and antioxidant defenses, (a) Recognition of parasites by host leukocytes (such as macrophages, neutrophils and eosinophils) occurs by pattern recognition receptors (PRR) that bind to pathogen-associated molecular patterns (PAMPs), or through antibodies (Ig), and leads to activation of host immune cells. Upon activation, these cells produce superoxide $\left(\mathrm{O}_{2}^{-}\right)$and nitric oxide $\left({ }^{\circ} \mathrm{NO}\right)$ radicals. $\mathrm{NO}$ is produced in the cytosol (but can cross membranes) by inducible nitric oxide synthase (iNOS); $\mathrm{O}_{2}^{-}$is produced by a multi-component, membrane-associated NADPH oxidase. Superoxide is released towards the extracellular space in the case of non-phagocytosable parasites (e.g., worms), or towards the phagosome (topologically equivalent to the extracellular space) in the case of intracellular parasites (e.g., protozoans). (b) ${ }^{\circ} \mathrm{NO}$ and ${ }^{\circ} \mathrm{O}_{2}{ }^{-}$react at diffusible controlled rate to produce peroxynitrite $\left(\mathrm{ONOO}^{-}\right)$. Peroxynitrite can react in one-electron oxidations (e.g., with transition metal centers), two electrons oxidations (of a given target), or with $\mathrm{CO}_{2}$, redirecting its reactivity. It also decomposes spontaneously into other ROS and RNS such as ${ }^{\circ} \mathrm{OH}$ and ${ }^{\circ} \mathrm{NO}_{2}$. In addition, activated neutrophils and eosinophils release myeloperoxidase and eosinophil peroxidases, respectively, which catalyze the conversion of hydrogen peroxide and halides into hypohalous acids. (c) Collectively, these products can inactivate enzymes, damage membranes and nucleic acids, and ultimately kill the parasitic organisms. (D) Parasites' defenses include antioxidant enzymes that directly scavenge superoxide, decreasing peroxynitrite formation (superoxide dismutases), and hydrogen and organic peroxide reductases (GPx and TPx). Some TPx have also been shown to reduce peroxynitrite catalytically. Repair mechanisms include methionine sulfoxide reductase, thioredoxin, and sulfiredoxin among others. * $\mathrm{R}$ 'H denotes a hydrocarbon chain, or alcohol $(\mathrm{R}$ ' $\mathrm{H}=$ $\mathrm{ROH})$, or a thiol $\mathrm{R}^{\prime} \mathrm{H}=\mathrm{RSH}$ ) 
with the highest levels present in the adult worm [8], the stage most resistant to oxidative stress and immune elimination [9]. In addition, GPx expression is upregulated by hydrogen peroxide and xanthine/xanthine oxidase generated ROS [10].

Recently, a search for GPx in Expressed Sequence Tag databases (dbEST) of platyhelminths identified a second GPx (GPx2) in S. mansoni and S. japonicum [11]. GPx2 also encodes a Sec residue at the active site and possesses an N-terminal signal peptide, which targets this isoform to the extracellular compartment, suggesting that this secreted variant would be important for extracellular hydroperoxide removal, helping to protect the parasite in its immediate environment. In this study, a GPx1 ortholog whose 3 '-untranslated region revealed the presence of a SECIS element was also identified in Echinococcus granulosus (another flatworm) transcriptome using the SECISearch algorithm (Chapter 9 and http://genome.unl.edu/SECISearch.html) [12].

In contrast to platyhelminths, the corresponding Cys-containing enzymes appear to occur in nematodes [13], as reviewed in [14]. Nevertheless, recent data-mining of nematode dbEST revealed some exceptions (see below) [15] Free-living nematode Caenorhabditis elegans has no Sec-containing GPx encoded in its genome [15].

\section{GSH- and Trx-reduction pathways in platyhelminth parasites are}

\section{controlled by a single selenoenzyme}

In most living organisms, there are two analogous and mutually supporting enzymic systems that provide antioxidant defense to cells: the glutathione (GSH) and the thioredoxin (Trx) systems (Figure 2) [16,17]. These systems have overlapping yet distinct targets. GSH, due to its reactivity and intracellular concentration, is one of the most important cellular antioxidants, being efficient in rescuing small disulfide molecules and in reacting directly with ROS. The major function of Trx is to maintain cysteine residues in substrate proteins in the reduced form. In addition to their direct function as antioxidants, GSH and Trx provide electrons to GPx and Trx peroxidase (TPx), respectively, which reduce hydrogen peroxide and organic hydroperoxides, and to methionine sulfoxide reductase, which is also an important antioxidant repair enzyme. GSH and Trx are usually reduced by GSH and Trx reductases (GR and TR), respectively, at the expense of NADPH oxidation.

Recent characterization of these systems in platyhelminth parasites has shown that 'conventional' GR and TR are absent; instead, the GSH and Trx systems are intermingled with the enzyme thioredoxin glutathione reductase (TGR), which provides reducing equivalents to both pathways (Figure 2).

(a) Comparison of the GSH, Trx and linked Trx-GSH systems

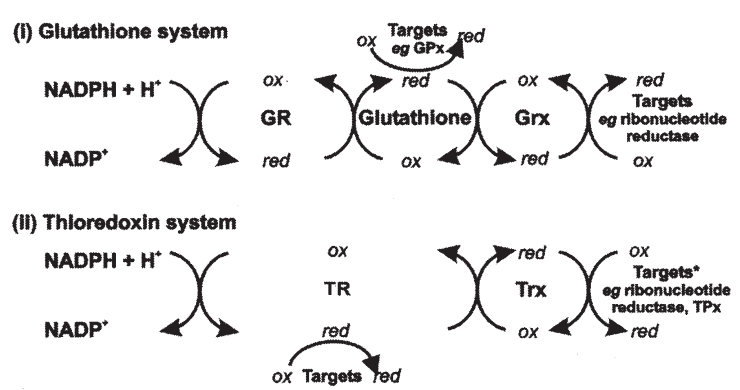

(III) LInked thloredoxin-glutathlone systems

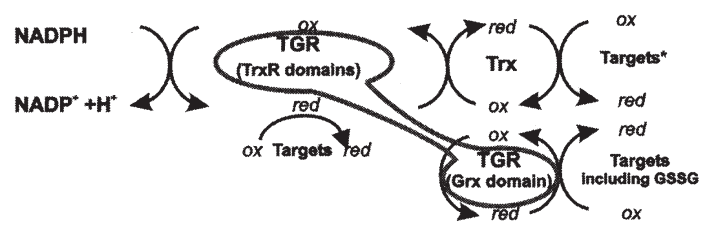

(b) Domains and redox centres in the primary structure of GR, TrxR, TGR, Grx and Trx

cxuxxc

GR
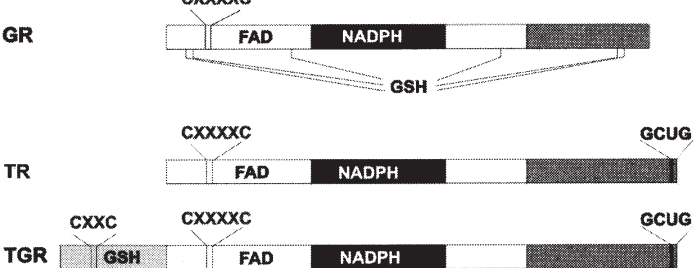

TGR

GSH

$\operatorname{cxxc}$

Trx एil

(c)

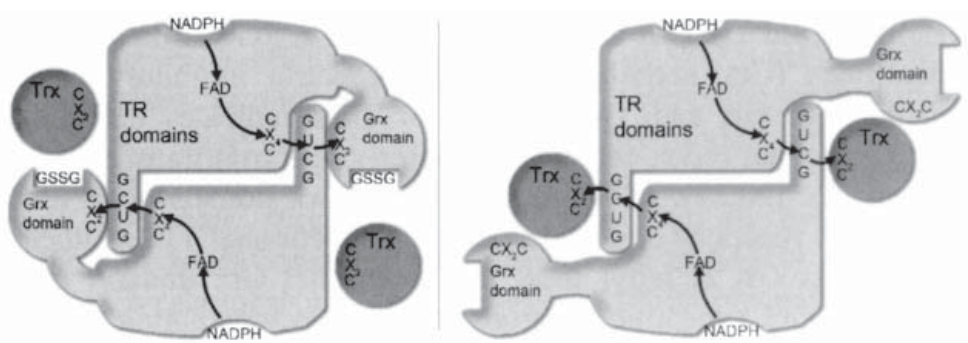

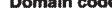

$\square$ domaln

domain

domain

GSH Grx domain

Trx domain

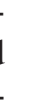


This protein is a second selenoenzyme family that has been characterized in platyhelminth parasites (reviewed in [11]). TGR is an oxidoreductase shown to possess TR, GR and Grx activities, achieving its broad substrate specificity by a fusion between Grx and TR domains (Figure 2b); this domain fusion was originally described in a mouse testis TGR [18].

Experimental and in silico data support the proposition that TGR is the single enzyme responsible for recycling both oxidized Trx and GSH in platyhelminth parasites. Treatment of $S$. mansoni adult worm extracts with auranofin, a known inhibitor of Sec-containing TRs, resulted in complete inhibition of TR and GR activities [19]. In addition, TGR was the single protein isolated from Taenia crassiceps (also a flatworm) extracts as a result of tracing GR and TR activities [20]. Examination of EST databases from Schistosoma species, which covers more than $90 \%$ of the gene content of this organism [21], revealed cDNAs encoding TGR, but not conventional TR or GR [11]. The biochemical characterization of E. granulosus and T. crassiceps TGR indicated that the native enzyme shuttles electrons from NADPH to oxidized Trx (TR activity), GSSG (GR activity) and glutathione-mixed disulfides (Grx activity). The stoichiometric inhibitory effect of auranofin on both GR and TR activities of TGR indicates that the Sec-containing C-terminal redox center participates in electron transfer to GSSG and oxidized Trx [20,22]. In addition, TR and Grx domains can function either in coupled reactions or independently. Conventional TRs neither bind GSH nor possess GR activity; thus, the N-terminal Grx domain of TGR would reduce GSSG, accepting electrons

Figure 2. Linked thioredoxin-glutathione systems, (a) Comparison of thioredoxin, glutathione and linked thioredoxin-glutathione systems. The glutathione system comprises (i) GR, GSH and Grx, whereas the thioredoxin system consists of (ii) TR and Trx. In linked Trx-GSH systems (iii), TGR functionally replaces TR, GR and Grx, providing reducing equivalents to targets of both systems. In all systems, NADPH is the upstream donor of reducing equivalents. (b) Components of the thioredoxin and glutathione systems. Redox centers of GR, TR, TGR, Grx and Trx are indicated, as well as the FAD prosthetic group and the ligands NADPH and GSH. TR and TGR possess a C-terminal extension missing in GR, which contains the C-terminal GCUG redox-active motif. TGR possesses an N-terminal Grx domain that is absent in TR and GR. The Grx and Trx domains contain the CXXC redox center. Grx, unlike Trx, binds GSH. (c) Schematic representation of electron flow in TGR. TGR, like GR and TR, is a homodimer, with monomers oriented in a head-to-tail manner. Electrons flow from NADPH to FAD, to the $\mathrm{CX}_{4} \mathrm{C}$ redox center, to the $\mathrm{C}$-terminal GCUG redox center of the second subunit, to the $\mathrm{CX}_{2} \mathrm{C}$ redox center of the Grx domain of the first subunit, and to targets, including GSSG (left scheme). Alternatively, electrons can flow, presumably directly, from the GCUG redox center to Trx (right scheme). The model proposes a flexible hinge, which connects the TR and Grx domains. This organization allows electrons to flow to the 'in built' Grx domain or to Trx. Parts (a) and (b) in the figure reprinted with modifications from [11] with copyright with permission from Elsevier. from the Sec-containing C-terminal redox center. The idea that the $\mathrm{C}$-terminal redox center donates electrons to the fused Grx domain implies that the Grx domain of TGR would be linked to the TR domains by a flexible hinge to allow reduction of the oxidized Trx (Figure 2c). It is interesting to note that $T$. crassiceps TGR showed a hysteretic behavior in enzymatic assays with GSSG at high concentrations; this observation led the authors to propose a model in which TGR would possess high and low affinity sites for glutathione [20]. Clearly, further biochemical characterization and structural data on this multifunctional enzyme are needed that will shed light on the mechanism of catalysis. In addition, molecular characterization of the corresponding gene could also provide clues regarding the mechanism of generation of isoforms. Indeed, the analysis of TGR in E. granulosus revealed two trans-spliced cDNAs derived from a single gene [22]. These variants code for mitochondrial (mt) and cytosolic (c) TGRs, containing identical Grx and TrxR domains, but differing in their N-termini. These variants derive from alternative initiation of transcription, followed by trans-splicing. Similarly, mtTGR and cTGR variants also derived from a single gene have been identified in S. mansoni [11].

Collectively, the results from platyhelminth studies strongly suggest that TGR is the main pyridine-nucleotide thiol-disulfide oxidoreductase in these organisms, in contrast to their hosts, where there is some redundancy of mechanisms for recycling oxidized Trx and GSH.

Very little has been published about these pathways in the other phylum of helminth parasites (Nematoda), and to the best of our knowledge, nothing is known about Sec/Cys-containing TR or TGR in parasitic nematodes. However, no single genome has yet been completed from metazoan parasites.

\section{Selenoproteins of nematode parasites: old families, unusual SECIS}

An in silico analysis of Caenorhabditis elegans and Caenorhabditis briggsae (free-living nematodes) genomes revealed that these organisms encode a single a selenoprotein, TR [15], corroborating earlier experimental data [23]. However, no experimental studies have yet been performed with Selenoproteins from parasitic nematodes. Nevertheless, in a recent study [15], the existing nematode ESTs were searched for selenoprotein genes using SECISearch and by screening for homologs of known Selenoproteins. These analysis identified selenoprotein homologs of selK, selT, selW, Sep15, selenophosphate synthetase and GPx. Two interesting points were noted from these analyses. First, various nematodes encode different selenoproteins, and the distribution of selenoprotein families within this phylum is mosaic. Second, it was found that all detected nematode selenoprotein genes contained an unusual form of SECIS element, with $G$ rather than a canonical $A$ at the conserved position preceding the quartet of non-Watson-Crick base pairs [15]. 


\section{Selenoproteins of protozoan parasites: waiting for surprises?}

Very little is known about selenoproteins from protozoan parasites. Recently, the presence of tRNA ${ }^{\mathrm{Sec}}$ was described in several species of the phylum Apicomplexa [24] (Lobanov et al., submitted). Plasmodium falciparum, which is the causative agent of malaria - the most overwhelming human parasitic infection, belongs to this phylum. The finding of tRNA ${ }^{\mathrm{Sec}}$ was consistent with the presence of putative EFsec and selenophosphate synthetase in $P$. falciparum and other Plasmodia. In addition, tRNA ${ }^{\mathrm{Sec}}$ was observed in Toxoplasma, but not in Cryptosporidium parasites. Genome-wide searches for SECIS elements in the six Plasmodium genomes revealed four selenoprotein genes. Interestingly, homology analyses of these proteins identified no hits outside Apicomplexa, suggesting that these selenoproteins do not exist in the apicomplexan hosts. These properties make the new selenoproteins attractive targets for anti-malaria drug development.

The other reference in the literature to a parasite Sec-decoding protozoan is the description of a Cys-containing selenophosphate synthetase from Leishmania major [25]. Leishmania belongs to trypanosomatidae family, which also includes Trypanosoma brucei, and T. cruzi (Table 1), which are causative agents of disabling and fatal diseases in the poorest rural population of the third world [26]. Consistent with the finding of selenophosphate synthetase, recent bioinformatics analyses revealed three selenoprotein genes in several Trypanosoma genomes (Lobanov and Gladyshev, unpublished).

Finally, no single reference could be found in the literature regarding a Sec-decoding amoebae, a traditional group of protozoa that include the parasitic amoebae of humans, Entamoebae histolytica.

\section{Parasite selenoproteins: drug or vaccine candidates?}

From a global perspective, the control of parasitic infections, which are a major cause of disability and mortality in many developing countries, remains as one of the most important challenges for medicine in the 21st century [2]. Although there are safe and effective drugs to control some parasitic diseases, parasites can develop resistance to drugs rendering them ineffective, as it has been the case of certain antimalarial drugs [27]. Thus, effective vaccines and new drugs against parasitic organisms are needed. The task ahead is enormous considering that parasite and hosts are eukaryotic organisms; as yet, there is not a single vaccine for a human parasitic infection. Whether selenoproteins can be drug targets or generate immunity depends on premises that are not necessarily different from those for any other target protein: the validity of a drug target would rely on it being an essential protein, and sufficiently different from the host homolog(s) as to be selectively inhibited. Likewise, a good vaccine candidate should generate an appropriate and selective immune response against the parasite, without inducing pathology to the host.
In platyhelminths, TGR is an attractive pharmacological target because of the lack of redundant mechanisms (i.e., TR and GR) to provide reducing equivalents to essential enzymes. Inhibition of this enzyme could lead to impaired synthesis of DNA and antioxidant defenses, compromising parasite survival. TGR may also be a good vaccine candidate, since it is a large protein with a degree of identity to host enzymes below $60 \%$. However, there are no studies regarding TGR as an immunogen. Contrary to TGR, there are promising studies on the use of GPx as a vaccine candidate. Vaccination of mice (not a natural host) against the platyhelminth $S$. mansoni with naked DNA constructs containing Sec-containing GPx showed significant levels of protection compared to a control group [28]. In this context, it is important to emphasize not only the fact that GPx appears to be important at the host parasite interface, but also that platyhelminth lack catalase and rely exclusively on GSH and Trx peroxidases for hydrogen peroxide removal.

In the case of protozoan parasites, further studies are needed to identify and functionally characterize their selenoproteins. Nevertheless, it is highly significant that the four selenoproteins identified in Plasmodium $s p$ have neither Sec nor Cys homologs in humans. Considering that Sec is usually located at the redox-active sites of enzymes, the selenol- and thiol-based redox systems may play vital an important role in the survival of protozoan parasites [29].

Finally, selenoproteins may be different to other proteins in one respect: electrophilic drugs, such as gold or platinum compounds, or alkylating agents that react preferentially with Sec over Cys may affect the parasite and the host to a different extent, depending on the relative importance of selenoproteins for the two organisms, and the presence/absence of Cys-containing enzymatic back up systems.

\section{Acknowledgements}

This work has been supported by Fogarty International Research Collaboration Award TW006959 and Ministry of Education, Uruguay, PDT 29/171.

\section{References}

1. K Warren 1988 The Biology of Parasitism PT Englund, A Sher (Ed) Alan R. Riss Inc New York 3

2. WHO The world health report - changing history. 2004 ( http://www.who.int/whr/2004/en/report04 en.pdf)

3. DH McGuinness, PK Dehal, RJ Pleass 2003 Trends Parasitol 19:312

4. BG Halliwell, JMC Gutteridge 1999 Free Radicals in Biology and Medicine Oxford University Press Inc New York

5. R Radi, G Peluffo, MN Alvarez, M Naviliat, A Cayota 2001 Free Radic Biol Mod 30:463 
6. DL Williams, RJ Pierce, E Cookson, A Capron 1992 Mol Biochem Parasitol 52:1277. M Maiorino, C Roche, M Kiess, K Koenig, D Gawlik, M Matthes, E Naldini, R Pierce, L Flohe 1996 Eur J Biochem 238:838

8. H Mei, PT LoVerde 1997 Exp Parasitol 86:69

9. GM Mkoji, JM Smith, RK Prichard 1988 Int J Parasitol 18:661

10. UE Zeick, B Von Janowsky 2004 Parasitology 128:493

11. G Salinas, ME Selkirk, C Chalar, RM Maizels, C Femandez 2004 Trends Parasitol 20:340

12. GV Kryukov, VM Kryukov, VN Gladyshev 1999 J Biol Chem 274:33888

13. L Tang, K Gounaris, C Griffiths, ME Selkirk 1995 J Biol Chem 270:18313

14. K Henkle-Duhrsen. A Kampkotter 2001 Mol Biochem Parasitol 114:129

15. K Taskov, C Chappie, GV Kryukov, S Castellano, AV Lobanov, KV Korotkov, R Guigo, VN Gladyshev 2005 Nucleic Acids Res 2005 33:2227

16. A Holmgren 2000 Antioxid Redox Signal 2:811

17. PG Winyard, CJ Moody, C Jacob 2005 Trends Biochem Sci 30:453

18. QA Sun, L Kimarsky, S Sherman, VN Gladyshev 2001 Proc Natl Acad Sci USA 2001 98:3673

19. HM Alger, AA Sayed, MJ Stadecker, DL Williams 2002 Int J Parasitol 32:1285

20. JL Rendon, IP del Arenal, A Guevara-Flores, A Uribe, A Plancarte, G Mendoza-Hernandez 2004 Mol Biochem Parasitol 133:61

21. S Verjovski-Almeida, R DeMarco, EA Martins, PE Guimaraes, EP Ojopi, AC Paquola, JP Piazza, MY Nishiyama, Jr., JP Kitajima, RE Adamson, PD Ashton, MF Bonaldo, PS Coulson, GP Dillon, LP Farias, SP Gregorio, PL Ho, RA Leite, LC Malaquias, RC Marques, PA Miyasato, AL Nascimento, FP Ohiweiler, EM Reis, MA Ribeiro, RG Sa, GC Stukart, MB Scares, C Gargioni, T Kawano, V Rodrigues, AM Madeira, RA Wilson, CF Menck, JC Setubal, LC Leite, E Dias-Neto 2003 Nat Genet 2003 35:148

22. A Agorio, C Chalar, S Cardozo, G Salinas 2003 J Biol Chem 2003 Apr 11 278: 12920

23. VN Gladyshev, M Krause, XM Xu, KV Korotkov, GV Kryukov, QA Sun, BJ Lee, JC Wootton, DL Hatfield 1999 Biochem Biophys Res Commun 259:244

24. T Mourier, A Pain, B Barrell, S Griffiths-Jones 2005 RNA 11:119

25. PC Jayakumar, VV Musande, YS Shouche, MS Patole 2004 DNA Seq 15:66

26. CM Morel, T Acharya, D Broun, A Dangi, C Elias, NK Ganguly, CA Gardner, RK Gupta, J Haycock, AD Heher, PJ Hotez, HE Kettler, GT Keusch, AF Krattiger, FT Kreutz, S Lall, K Lee, R Mahoney, A Martinez-Palomo, RA Mashelkar, SA Matlin, M Mzimba, J Oehler, RG Ridley, P Senanayake, P Singer, M Yun 2005 Science 309:401

27. TE Mansour Chemotherapeutic Targets in Parasites: Contemporary strategies $2002 \mathrm{~T}$ Mansour (ed) Cambridge University Press Cambridge 4

28. KA Shalaby, L Yin, A Thakur, L Christen, EG Niles, PT LoVerde 2003 Vaccine 22:130

29. S Muller, E Liebau, RD Walter, RL Krauth-Siegel 2003 Trends Parasitol 19:320 